ranges from 0 to 21 where higher values indicate worse $S Q$ ) of the Pittsburgh Sleep Quality Index. Linear regressions were performed to analyse the association of changes in ST and PA over time (predictor variables) with SQ at 2- and 5 -years follow-up (dependent variables) while considering baseline SQ, age, fat percentage, marital status, educational level, sleep or relaxation medication, and regular menstruation as confounders.

Results: Overall, after adjusting for confounders, non-statistical significant associations were found between changes in ST and PA intensity levels from baseline to 2-years follow-up with $S Q$ at 2-year follow-up $(P>0.05)$; except for the change in MVPA from baseline to 2-years follow-up, which showed evidence of statistical significance $(B=-0.207 ; P=0.059)$. Regarding the 5 -year follow-up, we did not observe either any association between changes in ST or PA intensity levels from baseline to 5-year follow-up with SQ at 5-year follow-up $(P>0.05)$.

Conclusion: The main findings suggest that neither ST nor PA intensity levels over time predict SQ at 2- and 5-year follow-up in women with fibromyalgia. Future PA-counselling randomised controlled trials might shed more light on the role that ST and PA could play on SQ.

References:

[1] Macfarlane GJ, et al. Ann Rheum Dis, 2017; 76(2):318-328.

[2] Borges-Cosic M, et al. Scand J Med Sci Sports 2019; 29:266-274.

Acknowledgments: This study was supported by the Spanish Ministry of Economy and Competitiveness (I+D+i DEP2010-15639; I+D+I DEP2013-40908-R) and the Spanish Ministry of Education, Culture and Sport (FPU15/00002).

Disclosure of Interests: : None declared

DOI: 10.1136/annrheumdis-2020-eular.4319

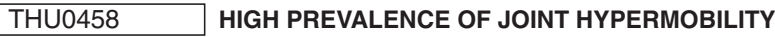 IN INFLAMMATORY BOWEL DISEASE PATIENTS WITH PAIN UNRESPONSIVE TO BOWEL-TARGETED} THERAPY

H. Zidany ${ }^{1}$, M. Waterman ${ }^{1,2}$, K. Toledano ${ }^{1,3}$, Y. Chowers $^{1,2}$, D. Markovits ${ }^{1,3}$, A. Karban ${ }^{1,4}$, A. Balbir-Gurman ${ }^{1,3}$, Y. Braun-Moscovici ${ }^{1,3} \cdot{ }^{1}$ Rappaport Faculty of Medicine, Technion, Haifa, Israel; ${ }^{2}$ Rambam Health Care Campus, Gastroenterology Institute, Haifa, Israel; ${ }^{3}$ Rambam Health Care Campus, $B$ Shine Rheumatology Institute, Haifa, Israel; ${ }^{4}$ Rambam Health Care Campus, Internal Medicine C, Haifa, Israel

Background: Musculoskeletal manifestations occur in $20-50 \%$ of patients (pts) with inflammatory bowel disease (IBD). A substantial number of patients complain of non-inflammatory musculoskeletal pain.

Objectives: To assess the incidence of joint hypermobility (JHM), benign joint hypermobility syndrome (BJHS) among patients with inflammatory bowel disease (IBD) examined in the inter-disciplinary rheumatology service at a tertiary referral center and the impact on IBD manifestations and outcome.

Methods: Medical records of 180 consecutive IBD pts referred to the interdisciplinary clinic were retrospectively reviewed. Data regarding age, gender, diagnosis, disease duration, clinical and laboratory features, previous and current therapy, Harvey-Brandshaw Index were entered into a database and analyzed. Beighton's scoring of $\geq 4 / 9$ was used to define patients with JHM. The 1998 Brighton's criteria were used to identify patients with BJHS. Outcome was defined as improvement of joint pain. The statistical methods used included descriptive statistics, T test, Spearman's correlation and multiple logistic regression analysis.

Results: Forty-six patients (mean(SD) age 36.2(12.4), disease duration $13.9(8.8)$ years) out of 180 IBD patients (mean(SD) age 40.4(14.3), disease duration 15.7(9.1) years) fulfilled the criteria for JHM. Twelve patients had active inflammatory joint disease ( 2 with axial involvement, 10 with peripheral joint disease and 2 with axial and peripheral joint involvement). The other 32 answered both major criteria for BJHS. The median Beighton scoring was 7 (range 5-9). Most of them were on biological treatment. Patients with JHM suffered frequently of arthralgia and abdominal pain, in spite of endoscopic remission and normal levels of calprotectin and inflammatory markers $(p=0.02, r=0.17)$. JHM and BJHS were associated with poorer outcome $(p=0.004, r=0.2)$. In a multiple logistic regression analysis, only JHM reached borderline significance for predicting worse outcome.

Conclusion: Joint and abdominal pain did not improve with immunomodulatory therapy in IBD patients with JHM. JHM may have a negative impact on achievement of clinical remission, in a significant subset of IBD patients. Rheumatologists and gastroenterologists should be aware of this.
Disclosure of Interests: : Haya Zidany: None declared, Matti Waterman: None declared, Kohava Toledano: None declared, Yehuda Chowers: None declared, Doron Markovits: None declared, Amir Karban: None declared, Alexandra Balbir-Gurman Consultant of: Novartis, Yolanda Braun-Moscovici: None declared DOI: 10.1136/annrheumdis-2020-eular.4767

\section{THU0459 CHRONIC MUSCULOSKELETAL PAIN AND CHRONIC WIDESPREAD PAIN IN CHILE: PREVALENCE AND ASSOCIATED FACTORS}

J. Duran ${ }^{1}$, P. Zitko ${ }^{1,2}$, P. Margozzini ${ }^{1} .{ }^{1}$ Pontificia Universidad Católica de Chile, Santiago, Chile; ${ }^{2}$ Universidad de Chile, Santiago, Chile

Background: Chronic musculoskeletal pain (CMP) is a key cause of health loss worldwide. Cultural factors may affect pain processing and it is key to have more information regarding CMP epidemiology in Latin America.

Objectives: We aimed to determine the prevalence of CMP and Chronic Widespread Pain (CWP) in Chile and to explore risk factors.

Methods: We used data recollected in the 2017 Chilean National Health Survey. Using COPCORD we defined CMP as non-traumatic pain for more than three months. CWP was defined by the presence of CMP in five body regions. Associations between CMP and CWP and risk factors was investigated through univariate and multivariate logistic regression models.

Results: After excluding subjects with missing information our final sample was 4045 subjects. CMP was present in $21,8 \%(95 \% \mathrm{Cl} 19,6 \%, 24,1 \%)$ and CWP in $4.2 \%(95 \% \mathrm{Cl} 3,3 \%, 5,1 \%)$. Significant risk factors for CMP in multivariate analysis were older age, female gender, lower educational level, and depressive symptoms. Protective factors for CMP were not being married and moderate alcohol consumption. CWP shared risk factors with CMP. (Table 1)

Conclusion: We found a high prevalence for CMP and CWP similar to values previously described. Female gender, older age, depressive symptoms and diabetes were the main risk factors associated with chronic pain, while moderate alcohol consumption was found to be protective.

References: $\mathrm{x}$

Disclosure of Interests: : None declared

DOI: 10.1136/annrheumdis-2020-eular.6692

THU0460

PHYSICAL FITNESS AND QUALITY OF LIFE IN WOMEN WITH FIBROMYALGIA: LONGITUDINAL ANALYSES FROM THE AL-ÁNDALUS PROJECT

B. Gavilán Carrera ${ }^{1,2}$, I. C. Alvarez-Gallardo ${ }^{1,3}$, M. Borges Cosic $^{1,2}$, A. Soriano Maldonado ${ }^{4,5}$, M. Delgado-Fernández ${ }^{1,2}$, V. Segura-Jiménez ${ }^{3}$. ${ }^{1}$ Faculty of Sport Sciences, University of Granada, Department of Physical Education and Sports, Granada, Spain; ${ }^{2}$ Sport and Health University Research Institute (iMUDS), Granada, Spain; ${ }^{3}$ Faculty of Education Sciences. University of Cádiz, Department of Physical Education, Puerto Real, Spain; ${ }^{4}$ Faculty of Education Sciences, University of Almería, Almería, Department of Education, Almeria, Spain; ${ }^{5}$ SPORT Research Group (CTS-1024), CERNEP Research Centre, University of Almería, Almeria, Spain

Background: Optimizing the highly deteriorated quality of life (QoL) of patients with fibromyalgia is one of the main goals in the management of the disease ${ }^{1}$ Physical fitness has been identified as a powerful marker of health that is positively related to QoL in this population ${ }^{2}$, although previous evidence is mainly based on cross-sectional data.

Objectives: This study aimed to examine the longitudinal associations (2- and 5-year follow-up) between physical fitness and QoL in women with fibromyalgia. Methods: In this prospective cohort study, women diagnosed with fibromyalgia (age: $51.3 \pm 7.6$ years) with completed data were included at baseline $(n=441)$, at 2-year follow-up $(n=220)$ and at 5-year follow-up $(n=227)$. The Senior Fitness Tests battery was used to assess physical fitness components and a standardized global fitness index was calculated. The eight dimensions plus the two physical and mental component summaries of the Short-Form health survey-36 questionnaire were used to assess QoL. To examine whether changes in fitness predicted QoL at follow-up, multiple linear regression models were built. The bidirectionallity of the associations (whether changes in QoL predicted fitness at follow-up) was also tested. Outcome values at baseline and age, fat percentage, analgesic consumption, educational level, and occupational status at follow-up were entered as potential confounders in all analyses.

Results: Changes in fitness were associated with physical function $(\beta=0.160)$, physical role $(\beta=0.275)$, bodily pain $(\beta=0.271)$, general health $(\beta=0.144)$, and 